Disclosure(s) Nothing to disclose.

\section{P114 POPULATION PHARMACOKINETICS AND DOSING OPTIMIZATION OF CEFEPIME IN NEONATES}

W Zhao*, TINN-Global Study Group. Shandong University, Jinan, China

\subsection{6/archdischild-2019-esdppp.152}

Objective Cefepime, a fourth-generation cephalosporin, is used in the treatment of severe nosocomial infections in neonates. Pharmacokinetics of cefepime was limited. Therefore, we aimed to study the population pharmacokinetics of cefepime and optimize cefepime regimen in preterm and term neonates. Methods Blood samples were obtained from neonates treated with cefepime using an opportunistic sampling design. Concentration of cefepime was determined by high performance liquid chromatography. Population pharmacokinetics analysis was conducted using NONMEM software.

Results Sparse pharmacokinetic samples $(n=100)$ from 85 neonatal patients were available for analysis. A one-compartment model with first-order elimination was used to describe the pharmacokinetics of cefepime. Covariate analysis showed that current weight, postmenstrual age and serum creatinine concentration had tremendous influence on pharmacokinetics of cefepime. Monte Carlo simulation indicated that current dosage regimen $(30 \mathrm{mg} / \mathrm{kg}, \mathrm{q} 12 \mathrm{~h}$ ) was correlated with high risk of underdosing in neonates. To achieve the target rate of $70 \%$ of patients get free drug concentration above MIC during $70 \%$ of dosing interval, $30 \mathrm{mg} / \mathrm{kg} \mathrm{q} 8 \mathrm{~h}$ was required for all neonates, using susceptibility breakpoint of $4 \mathrm{mg} / \mathrm{L}$.

Conclusion The population pharmacokinetics characteristics of cefepime were evaluated in neonates. Based on simulation, different dosage regimens were required depending on the postmenstrual age and pathogens.

Disclosure(s) Nothing to disclose.

\section{P115 EFFECTS OF THE CLINICAL PHARMACIST'S INTERVENTION ON RATIONALITY OF PARENTERAL NUTRITION}

M Zhu*, J Xu. Nanjing Children's Hospital, Nanjing, China

10.1136/archdischild-2019-esdppp.153

Objective Through STRONGkids ${ }^{1}{ }^{2}$ (screening tool risk on nutritional status and growth of children) to observe the influences on nutritional indicators and postoperative recovery of different nutritional risk levels of children with intussusception in the use of parenteral nutrition support. Through educating and interventing the doctors to promote the use of parenteral nutrition more reasonable and the hospitalization costs lower. ${ }^{3}$ Methods Children were grouped according to different scores of STRONGkids, ${ }^{4} 5$ and each group was divided into two groups $\mathrm{A}$ and $\mathrm{B}$ according to using parenteral nutrition only or no nutrition support at all. The proportion of the two groups, nutritional indicators and postoperative recovery of the children after surgery were compared to observe the parenteral nutrition usage rate of different groups, and the use of parenteral nutrition was necessary or not. The clinical pharmacist intervened the doctors according to the research results. 1 year later, the indicators above were compared again.
Results There were no significant differences on nutritional indicators and postoperative recovery in 1-2 score groups between group $\mathrm{A}$ and $\mathrm{B}$, but the hospitalization cost in group A was significantly higher than that in group B. In 3-score group of children, the decreases of weight, prealbumin and retinol binding protein were more significant in group $B$ than in group A, and the hospitalization days of group A were significantly shorter than group B. The incidence of adverse reactions of using parenteral nutrition was significantly higher. According to above results, the clinical pharmacist instructed doctors to improve the indication of parenteral nutrition according to the relevant guidelines. 1 year later, the usage rate of parenteral nutrition dropped in 1-2 score groups. The incidence of adverse reactions and the costs of hospitalization were significantly decreased.

Conclusions The clinical pharmacist played an important role in promoting the rational use of parenteral nutrition. ${ }^{6} 7$

\section{REFERENCES}

1. Teixeira AF, Viana KD.Nutritional screening in hospitalized pediatric patients: a systematic review.[J]J Pediatr (Rio J) 2016, 92(4):343-352.

2. Forga L, Bolado F, Goñi MJ,et al. Low serum levels of prealbumin, retinol binding protein, and retinol are frequent in adult type 1 diabetic patients.J Diabetes Res 2016;2016:2532108. doi: 10.1155/2016/2532108. Epub 2016 Nov 29.

3. Pediatric Collaborative Group, Society of Parenteral and Enteral Nutrition. Guidelines for pediatric clinical application of enteral and parenteral nutritional support in China[J]. Zhonghua Er Ke Za Zhi, 2010, 48(6):436-441.

4. Abunnaja S, Cuviello A, Sanchez JA.Enteralandparenteral nutritionin the perioperative period: state of the art[J].Nutrients. 2013, 5(2):608-623.

5. Yi F, Ge L, Zhao J, Lei Y,et al.Meta-analysis:total parenteral nutritionversustotalenteral nutritionin predicted severe acute pancreatitis[J].Intern Med. 2012, 51 (6):523-530.

Disclosure(s) Nothing to disclose.

\section{\begin{tabular}{l|l} 
P116 PROPHYLACTIC USE OF ENOXAPARIN DURING \\
\hline
\end{tabular} BARIATRIC SURGERY IN ADOLESCENTS WITH SEVERE OBESITY}

$1,2^{2}$ Z Ziesenitz ${ }^{*},{ }^{3}$ J Vaughns, ${ }^{4}$ E Williams, ${ }^{5} \mathrm{E}$ Nadler, ${ }^{6} \mathrm{G}$ Mikus, ${ }^{1,4} \mathrm{~J}$ van den Anker. ${ }^{1}$ Pediatric Pharmacology and Pharmacometrics, University Children's Hospital Basel (UKBB), Basel, Switzerland; ' 2 Pediatric Cardiology, University Children's Hospital Heidelberg, Heidelberg, Germany; ${ }^{3}$ Anesthesia and Pain Medicine and Clinical Pharmacology; ${ }^{4}$ Clinical Pharmacology; ${ }^{5}$ Surgery, Children's National Health System, Washington, DC, USA; ${ }^{6} \mathrm{Clinical}$ Pharmacology and Pharmacoepidemiology, Universty Hospital Heidelberg, Heidelberg, Germany

\subsection{6/archdischild-2019-esdppp.154}

Background Severe obesity predisposes adults and youth to a higher risk of venous thromboembolism (VTE). Enoxaparin is frequently used for their VTE management. This study evaluates a BMI-stratified prophylactic dosing regimen of enoxaparin in severely obese adolescents undergoing bariatric surgery.

Methods This prospective study enrolled severely obese adolescents aged 12-20 years undergoing laparoscopic sleeve gastrectomy. Prophylactic enoxaparin was dosed at $40 \mathrm{mg}$ SC (for a BMI less than $50 \mathrm{~kg} / \mathrm{m}^{2}$ ) and $60 \mathrm{mg}$ SC (for a BMI equal to or greater than or $50 \mathrm{~kg} / \mathrm{m}^{2}$ ). Blood samples were drawn until 12 hrs post-dose. Plasma Anti-Factor Xa (Anti-FXa) activity was used as a surrogate marker for enoxaparin plasma concentration and pharmacokinetics were assessed using non-compartmental PK analysis. The primary efficacy outcome was the anti-FXa activity 4-6 hours after dosing, and the primary endpoint was the proportion of patients who reached prophylactic anti-FXa activity of $0.1-0.3 \mathrm{U} / \mathrm{mL}$ between $4-6$ hours after dosing. 
Results Ten female and two male obese adolescents (age range 14-19 years) had a mean body weight of $140.8 \mathrm{~kg}$ (93.7-174 $\mathrm{kg}$ ) and a mean BMI of $49.9 \mathrm{~kg} / \mathrm{m}^{2}\left(38.4-58 \mathrm{~kg} / \mathrm{m}^{2}\right)$. Four patients received $40 \mathrm{mg}$ enoxaparin, 8 patients were dosed with $60 \mathrm{mg}$ enoxaparin. No VTE or major bleeding occurred. Peak plasma anti-FXa activity $\left(\mathrm{C}_{\max }\right)$ ranged from $0.14-0.30$ $\mathrm{IU} / \mathrm{mL}$ (median $\mathrm{C}_{\max } 0.205 \mathrm{IU} / \mathrm{mL}$ ). Median $\mathrm{T}_{\max }$ was 5.67 hours (range 3.78-7.52 hours). Median $\mathrm{AUC}_{\mathrm{i}}$ was $1.00 \mathrm{~h}$ *IU/ $\mathrm{mL}$ (range $0.42-1.67 \mathrm{~h} / \mathrm{IU} / \mathrm{mL}$ ). 10 out of 12 patients $(83 \%)$ reached the primary endpoint with anti-FXa activity in the range for VTE prevention $(0.1-0.3 \mathrm{IU} / \mathrm{mL})$.

Conclusions In this single center cohort study, the dosing scheme of $40 \mathrm{mg}$ vs $60 \mathrm{mg}$ enoxaparin stratified according to $\mathrm{BMI}$ proved to be effective in reaching prophylactic anti-FXa activity in $83 \%$ of adolescent patients. This dosing scheme is in accordance with current practice in adults.

Disclosure(s) J. Vaughns and J. van den Anker are supported by the Eunice Kennedy Shriver National Institute of Child Health and Development (5T32HD087969).

\section{P117 DOSE EVALUATION OF INTRAVENOUS METAMIZOLE (DIPYRONE) IN INFANTS AND CHILDREN: A PROSPECTIVE POPULATION PHARMACOKINETIC STUDY}

${ }^{1} \mathrm{~V}$ Ziesenitz* 1,2 $\mathrm{F}$ Rodieux ${ }^{1,3} \mathrm{~A}$ Atkinson, ${ }^{1} \mathrm{C}$ Borter, ${ }^{1,4} \mathrm{~J}$ Bielicki, 5,6 M Haschke, ${ }^{5} \mathrm{U}$ Duthaler, ${ }^{5} \mathrm{~F}$ Bachmann, ${ }^{7} \mathrm{~T}$ Erb, ${ }^{8} \mathrm{~N}$ Gürtler, ${ }^{9} \mathrm{~S}$ Holland-Cunz, ${ }^{1,10 \mathrm{~J}}$ van den Anker, ${ }^{1} \mathrm{~V}$ Gotta, ${ }^{1} \mathrm{M}$ Pfister. 'Pediatric Pharmacology and Pharmacometrics, University Children's Hospital Basel (UKBB), Basel; '2Division of Clinical Pharmacology and Toxicology, Department of Anesthesiology, Pharmacology, Intensive Care and Emergency Medicine, Geneva University Hospitals, Geneva; ${ }^{3}$ Department of Infectious Diseases, University Hospital Bern, Bern; ${ }^{4}$ Division of Pediatric Infectious Diseases, University Children's Hospital Basel (UKBB); ${ }^{5}$ Clinical Pharmacology and Toxicology, Department of Biomedicine and Clinical Research, University and University Hospital of Basel, Basel; ${ }^{6}$ Clinical Pharmacology and Toxicology, Department of General Internal Medicine, University Hospital Bern, Bern; ${ }^{7}$ Pediatric Anesthesiology, University Children's Hospital Basel (UKBB); ${ }^{8}$ Otolaryngology, Head and Neck Surgery, University and University Hospital of Basel; ${ }^{9}$ Pediatric Surgery, University Children's Hospital Basel (UKBB), Basel, Switzerland; ${ }^{10}$ Clinical Pharmacology, Children's National Health System, Washington, DC, USA

\subsection{6/archdischild-2019-esdppp.155}

Background The prodrug metamizole is frequently dosed intravenously (IV) for postoperative pain in children of all ages, despite its off-label use in infants $<1$ year. We aimed to investigate the pharmacokinetics (PK) of the main metabolite of metamizole, 4-aminoantipyrine (MAA), in children aged 372 months following IV dosing.

Methods $10 \mathrm{mg} / \mathrm{kg}$ metamizole was administered IV for postoperative analgesia. PK samples were drawn at 5 predefined time points. PK of the main active metabolite MAA and three other metabolites was characterized by both non-compartmental (NCA) and population PK analysis (PPK). $\mathrm{AUC}_{0 \text {-inf }}$ of MAA was calculated by NCA for two age cohorts (3-23 months, 2-6 years) and compared to the $80-125 \%$ range of adult dose-adjusted reference exposure $\left(\mathrm{AUC}_{\mathrm{ref}}\right)$. PPK investigated age and weight dependency of the kinetics, and dosing strategies to achieve equivalent adult exposure in children.

Results A total of 25 children aged 5 months - 5.8 years $(7.8-24.8 \mathrm{~kg})$ with at least one plasma concentration sample were included in PPK, 19 children who had 5 predefined samples up to $10 \mathrm{~h}$ post-dose were included in NCA. $\mathrm{AUC}_{0-}$ inf of MAA in children of 2-6 years was 29.8 (95\%CI 23.338.1) $\mathrm{mg} / \mathrm{L} * \mathrm{~h}$, significantly lower than $\mathrm{AUC}_{\mathrm{ref}}(80 \%-125 \%$ range: $\left.39.2-61.2 \mathrm{mg} / \mathrm{L}^{*} \mathrm{~h}\right)$. $\mathrm{AUC}_{0 \text {-inf }}$ of $\mathrm{MAA}$ in infants of 3-23 months was 42.5 (95\%CI 15.7-115.4) $\mathrm{mg} / \mathrm{L} * \mathrm{~h}$, overlapping with $\mathrm{AUC}_{\text {ref. }}$ The large variability observed in infants could be partially explained by covariates body weight and age.

Conclusions Kinetics of the main active metabolite MAA depends on age in infants and children. MAA exposure after a single IV dose of $10 \mathrm{mg} / \mathrm{kg}$ metamizole in infants $<1$ year of age was higher compared to an equal dose in adults and older children. This suggests that the optimal dose for this age group to achieve equivalent exposure compared to adults is lower than currently recommended.

Disclosure(s) Nothing to disclose.

\section{P118 'MEDICINES FOR CHILDREN' PROJECT: PUTTING FAMILIES AND CARERS IN THE CENTRE}

${ }^{1} \mathrm{E}$ Zolotas*, ${ }^{2} \mathrm{H}$ Sammons, ${ }^{3} \mathrm{D}$ Tuthill, ${ }^{4} \mathrm{C}$ Barker, ${ }^{5} \mathrm{~S}$ Tomlin, ${ }^{6} \mathrm{~L}$ Partridge, ${ }^{7} \mathrm{~A}$ Rossiter, ${ }^{7} \mathrm{~V}$ Osmond, ${ }^{8} \mathrm{H}$ Barham, ${ }^{9} \mathrm{~A}$ Fox, Medicines For Children Project Board. ${ }^{1}$ Paediatrics, Royal Derby Hospital, Derby,; ${ }^{2}$ University of Nottingham, Nottingham,; ${ }^{3}$ University Hospital of Wales, Cardiff; ; ${ }^{4}$ Alder Hey Children's NHS Foundation Trust, Liverpool,; ${ }^{5}$ Great Ormond Street Hospital, London,; ${ }^{6}$ Wellchild, Cheltenham,; ${ }^{7}$ Royal College of Paediatrics and Child Health, London,; ${ }^{8}$ The Text Doctor, Oxford,; ${ }^{9}$ University Hospital Southampton NHS Foundation Trust, Southampton, UK

\subsection{6/archdischild-2019-esdppp.156}

Background 'Medicines for Children' (MfC) is a joint initiative between the children's charity WellChild, the Royal College of Paediatrics and Child Health and the Neonatal and Paediatric Pharmacists Group. The project aim is to provide parents and carers with reliable, accurate and accessible information about their child's medicines.

Methods In 2006, 600 parents and carers were surveyed in order to understand what information was needed. Paediatricians, pharmacists and a medical editor then liaised with WellChild to develop a leaflet template and wrote a set of pilot leaflets. The leaflet production was subsequently standardised. An eight-step process is followed including consultation with health professionals, families and carers. The leaflet library and a series of information videos has grown, with the assistance of a dedicated group of volunteer authors. Published leaflets are reviewed every three years. Access to information is free of charge. The project is funded by the 3 partner groups and not by pharmaceutical companies.

Results MfC hosts over 230 leaflets and videos. The MfC website $^{1}$ was launched in 2009. It was subsequently reviewed by parents and carers and re-developed in 2011 and 2015. MfC has users accessing the site from every country in the world. The information leaflets have been viewed over 3 million times in 2018, up from 7,200 in 2009. In 2014, an independent audit of MfC found that over $90 \%$ of the parents surveyed thought that the leaflets had an appropriate layout and conveyed the information in lay terminology. Since 2011, MfC information leaflets have been certified by National Health Service Information Standard as providing high quality health information for the public.

Conclusion MfC is a successful and acclaimed project which provides high quality, reliable and accurate medicines information worldwide for more than a decade.

\section{REFERENCE}

1. https://www.medicinesforchildren.org.uk/.

Disclosure(s) Nothing to disclose. 